\title{
Tecnura
}

\section{Identificación de malas prácticas constructivas en la vivienda informal. Propuesta educativa}

\section{Identification of constructive bad practices in informal housing. Educational proposal}

\author{
Camilo Alberto Torres Parra' ${ }^{1}$ Janneth Arias Hernandez ${ }^{2}$
}

Fecha de recepción: 02 de agosto 2018

Fecha de aceptación: 12 de noviembre de 2018

Cómo citar: Torres P., C.A. y Arias H., J. (2019). Identificación de malas prácticas constructivas en la vivienda informal. Propuesta educativa. Tecnura, 23(59), 47-59. DOI: https://doi.org/10.14483/22487638.14823

\section{Resumen}

Contexto: Este trabajo presenta una propuesta educativa y participativa, liderada por la Red de Cooperación Construya Seguro y Sostenible, a la cual pertenece la Corporación Universitaria Minuto de Dios, la Universidad Católica de Colombia y la Universidad Piloto de Colombia desde sus programas de Ingeniería Civil.

Metodología: Esta investigación se desarrolló a partir de un método cuantitativo descriptivo no experimental, en donde se identificaron cuarenta y cuatro (44) malas prácticas ligadas a la habitabilidad en la vivienda informal.

Resultados: Estas malas prácticas se tradujeron en objetivos instruccionales mediante unidades de aprendizaje enmarcadas en una propuesta de curso orientado a comunidades bajo el modelo instruccional de Jerrold y Kemp.

Conclusión: Esta iniciativa permitió vincular a 25 familias del barrio Villas de Kennedy, en Bogotá (Colombia), con estudiantes y docentes vinculados a la Red, quienes mediante un trabajo participativo socializaron sus hallazgos y experiencias.

Palabras clave: vivienda informal, habitabilidad, ingeniería, educación.
Abstract

Context: This paper presents a participative educational proposal led by the cooperation network caIled "Construya Seguro y Sostenible," to which the University Corporation Minuto de Dios, the Catholic University of Colombia, and the Pilot University of Colombia belong.

Methodology: This research was developed from a non-experimental descriptive quantitative method, where forty-four (44) bad practices related to habitability in informal housing were identified.

Results: These bad practices were translated into instructional objectives through learning units framed in a course proposal oriented to communities under the instructional model of Jerrold and Kemp.

Conclusion: This initiative allowed to link 25 families of the Villas de Kennedy neighborhood in Bogotá, Colombia, with students and teachers linked to the Network who, through participatory work, socialized their findings and experiences.

Keywords: Informal housing, habitability, engineering, education.

1 Ingeniero Ambiental y Sanitario, especialista en Gerencia de Proyectos, magister en Educación. Docente de la Universidad Católica de Colombia. Bogotá, Colombia. Contacto: catorres@ucatolica.edu.co. ORCID: https://orcid.org/0000-0003-0431-1191

2 Trabajadora Social, magister en Antropologia Social y Educación. Docente de la Corporación Universitaria Minuto de Dios. Bogotá, Colombia. Contacto: jnarias@uniminuto.edu. ORCID: https://orcid.org/0000-0001-8143-6495 


\section{INTRODUCCIÓN}

La mayoría de las familias latinoamericanas aspiran a ser dueñas de su vivienda y muchos de los gobiernos estimulan esa aspiración desde políticas asistencialistas, ya sea mediante el ofrecimiento de subsidios o la permisividad de tenencia de tierra en donde las técnicas constructivas utilizadas se caracterizan por la autoconstrucción no regulada ni asesorada, escenario que pone en riesgo el bienestar de los individuos que residen en estas.

En este sentido, la vivienda como un espacio físico no resulta en todos los casos ser garante de un mejor nivel de vida, debido a que en sí misma puede no proporcionar las mínimas condiciones de seguridad, salubridad, higiene, calidad y espacio requerido para que uno o varios individuos desarrollen su proyecto de vida sin afectar su integridad física y emocional.

En varias regiones de Colombia se presentan problemas asociados a la vivienda, como los mencionados anteriormente, razón por la cual el estudio propuesto en este documento surge a través de Swisscontact, una fundación orientada a promover el desarrollo económico, social y ambiental generando oportunidades productivas, educativas y empresariales para que las personas puedan mejorar sus condiciones de vida mediante su propio esfuerzo. Dicha institución, en su capítulo colombiano y por medio de sus profesionales y redes de cooperación, es donde se ha ejecutado el proyecto "Promoción de prácticas de construcción sostenible en sectores urbanos vulnerables".

El proyecto tiene por objetivo mejorar las condiciones de calidad de vida de la población en las principales áreas urbanas informales de Colombia y reducir las malas prácticas constructivas en las unidades habitacionales, a través de la capacitación de la mano de obra empírica que realiza estos trabajos.

La meta principal de esta fundación es el desarrollo de currículos que involucren prácticas sostenibles, seguras y saludables de construcción y, a partir de ellos, realizar la formación para mejorar las destrezas de maestros de obra, auxiliares de la construcción, ferreteros y población propietaria en las zonas vulnerables.

Teniendo en cuenta la anterior experiencia, se tomó como centro de acción para ejecutar la evaluación técnica y la propuesta educativa, la localidad de Kennedy, la cual presenta variados problemas en torno a la habitabilidad. Posee un déficit de vivienda elevado (11.493 unidades), está afectada por la presencia de depósitos no regulados de residuos propios de la actividad antrópica en $13,7 \%$, y los inquilinatos y cuartos están entre un rango de $0,5 \%$ a 1,2 \% en relación con los apartamentos $(62,4 \%)$ y casas $(36 \%)$. Las unidades habitacionales, en $35 \%$, no poseen una adecuada ventilación, sus materiales están en mal estado, la seguridad estructural es baja y las condiciones de habitabilidad son precarias para sus habitantes (Universidad Javeriana, 2012).

En función de lo anterior, este documento se estructura de la siguiente manera: a) se presentan algunos cuestionamientos frente a la situación de habitabilidad e informalidad en la vivienda presentes en la población objeto de estudio, los cuales se abordan y se resuelven a la luz del marco teórico planteado. b) Se sugiere un marco teórico en el que se explica lo que se entiende por habitabilidad, vivienda informal y sus malas prácticas constructivas, así como la importancia y las implicaciones de un diseño educativo instruccional. c) Se muestra el proceso metodológico que se compone de la propuesta educativa enfocada a la comunidad y se propone una metodología de formación social participativa que se encarga de promover la articulación de los saberes y experiencias de las comunidades con los conocimientos de los estudiantes y docentes de las universidades. d) Se exponen los resultados. e) Se detallan los principales aprendizajes y conclusiones que se derivan de la propuesta educativa constructivista y sugerencias para trabajos futuros.

Teniendo en cuenta la situación de habitabilidad e informalidad en la vivienda presente en la población objeto de estudio, surgieron las 
siguientes preguntas de investigación: ¿Cómo diseñar un curso libre participativo para comunidades que habitan en viviendas informales que presentan malas prácticas constructivas en habitabilidad?; ¿cómo transmitir el conocimiento recabado en campo con estudiantes de ingeniería en relación a las malas prácticas constructivas de habitabilidad?; ¿qué elementos debe tener una propuesta constructivista en torno a las buenas prácticas constructivas, que contribuya al mejoramiento de las condiciones de habitabilidad en la vivienda de la comunidad? Para responder a estos interrogantes fue necesario abordar dicho contexto desde el aspecto educativo, pues este permite que las personas interpreten su experiencia en función de sus propios conocimientos, ya que todo cambio en la organización cognitiva del estudiante es una construcción personal a partir de las experiencias de aprendizaje y la realidad presente de cada quien, según su contexto y territorio, en las que las personas mejoran sus capacidades y las amplían (Cubero, 2005).

En este sentido, la consolidación de una propuesta educativa socialmente responsable desde la ingeniería debe ser vista como la capacidad del individuo y de la comunidad académica de reconocer su realidad y la de otros, cuestionándola y analizando la relación causa/efecto y los intereses que le subyacen, y a partir de allí, construir un posicionamiento frente a ella. Precisamente una educación para el desarrollo humano es la que mantiene una relación directa con la investigación y la proyección social, buscando que el estudiante se vincule en procesos de transformación social que beneficien y propendan por el bienestar de poblaciones o grupos con algún grado de vulnerabilidad, bien sea por su condición social, económica, etaria, cultural o política (Morán, 2010).

Asimismo, se debe entender la importancia de estos espacios para la educación en ingeniería, porque dentro de la propuesta de formación las prácticas pedagógicas están direccionadas a que los estudiantes reconozcan la realidad de su entorno y construyan estrategias de acción por medio de las cuales participen e incidan en la transformación positiva de este. Para este logro, se debe contar con la capacidad de identificar las problemáticas que comprometen la calidad de vida de las poblaciones menos favorecidas, y hay que poner al servicio de la sociedad civil y del conocimiento la formación adquirida en el aula, para demostrar con este ejercicio pedagógico la importancia que tienen los contextos vulnerables para la ingeniería y viceversa. Así se estaría aportando a la formación de estudiantes éticos, responsables, y sensibles a los problemas de la sociedad.

\section{MARCO TEÓRICO}

\section{Habitabilidad}

La habitabilidad puede ser entendida como la capacidad de los espacios construidos para satisfacer las necesidades objetivas y subjetivas de las personas y núcleos familiares, por lo tanto, se deben tener en cuenta las relaciones físicas y psicosociales que se establecen en la unidad habitacional, las cuales van a permitir el sano desarrollo físico, biológico, psicológico y social de la persona, de ahí su estrecha vinculación con el concepto de calidad de vida y bienestar (Zulaica y Celemín, 2008).

Una de las problemáticas sentidas y diagnosticadas en los últimos años es el aumento progresivo de la habitabilidad precaria en el mundo; lo que ha agudizado la situación de los asentamientos urbanos y rurales en donde se encuentra que un tercio del planeta, 2240 millones de personas, carece de necesidades básicas de cobijo y 925 millones habitan en entornos perjudiciales para su salud. Asimismo, la Organización de las Naciones Unidas establece que aproximadamente 2000 millones de personas habitan en asentamientos urbanos precarios, por lo que las comunidades de esos sectores conviven en condiciones de informalidad, lo cual potencializa su vulnerabilidad, alojándose en viviendas temporales, con materiales de desecho y ubicadas en las periferias de las grandes urbes (ONU-Hábitat, 2014). 
Asimismo en Colombia, dadas las condiciones de desigualdad frente al ingreso, educación, ocupación y acceso a la vivienda, se dio lugar al surgimiento de alternativas informales en la habitabilidad, en especial las invasiones de terrenos y las urbanizaciones denominadas piratas, que permitieron a las familias de bajos recursos el acceso a una solución de vivienda parcial, inicialmente en condiciones muy precarias, pero motivadas por una urgencia de tener un techo que brindara protección y bienestar a sus familias Por esto , aproximadamente 3.828.055 hogares se encuentran en déficit a nivel nacional: 1.307.757 tienen déficit cuantitativo de vivienda, mientras que 2.520.298 tienen déficit cualitativo (Rueda, 2011).

Bogotá no es ajena a este panorama y dentro de sus 20 localidades, 13 presentan un panorama de vulnerabilidad en habitabilidad relacionado a los altos precios de la vivienda formal, la autoconstrucción y mano de obra no capacitada para edificar viviendas, materiales en mal estado, terrenos inseguros, ocupación desordenada del territorio y desconocimiento de los riesgos a los que se está expuesto, debido a la percepción subjetiva del habitante, en donde el conformismo es generalizado puesto que no se conoce otro entorno, y sus limitaciones económicas, educativas y de salud, los colocan en un nivel de riesgo alto en lo que frente a habitabilidad urbana se refiere (Escallón, 2012).

\section{Vivienda informal}

La vivienda informal es aquella que no se encuentra enmarcada en la normatividad técnica en relación con su calidad en los materiales, distribución de espacios, confort, seguridad estructural, ubicación y seguridad de tenencia, entre otros. Esta tipología de unidad habitacional se caracteriza frente a su edificación por procesos de autoconstrucción, mano de obra empírica y falta de documentación que acredite su tenencia. Estas situaciones afectan tanto a países desarrollados como en vía de desarrollo, presentándose esta problemática económica y social de acceso y calidad, cuando un país carece de mecanismos institucionales fuertes que generen las condiciones mínimas de habitabilidad para sus habitantes (4-Hábitat, 2012).

En ese sentido, el concepto de vivienda informal se asocia directamente a la habitabilidad precaria a nivel urbano, por eso es importante que las prácticas constructivas estén encaminadas a proporcionar a los moradores satisfactores de accesibilidad, movilidad, emplazamiento, espacio público, servicios públicos, de mobiliario urbano, de zonas verdes, y la disponibilidad de transporte público, entre otros. Por tales motivos, la vivienda informal debe relacionar su calidad constructiva con la normatividad legal vigente y la calidad en sus acabados y distribución de espacios, atendiendo al cumplimiento de normas mínimas de construcción que permitan habitar con confort y seguridad a sus núcleos familiares (Espinoza, Gómez, 2010).

Por consiguiente, mediante la promoción de las buenas prácticas constructivas en habitabilidad se busca disminuir la informalidad en la construcción de la vivienda. Se parte de comprender que con la capacidad de los espacios construidos para satisfacer las necesidades físicas y biológicas de los individuos hay estándares de calidad técnica que promueven la seguridad y el bienestar en la manera de diseñar y habitar un espacio común que permita un sano desarrollo de la persona (Zulaica y Celemín, 2008).

\section{Malas prácticas constructivas en la vivienda}

Colombia es un país que se ha caracterizado porque sus ciudades se han edificado históricamente bajo procesos informales constructivos, y dada una actividad sísmica media y terrenos con altas pendientes, el mayor riesgo catastrófico se centra en el sector de las viviendas de estratos socioeconómicos medios y bajos. Sus unidades habitacionales carecen de especificaciones sismorresistentes, es decir, de un diseño que propende por la apropiada configuración estructural, con componentes de dimensiones simétricas y materiales con una 
proporción y resistencia suficientes para soportar la acción de fuerzas causadas por sismos y la estabilización de un terreno (Asosísmica, 2001). Su diseño es concebido sin confort y la distribución de espacios no es óptima, lo que genera condiciones inaceptables de humedad, salubridad, iluminación, ruido y ventilación. Estos factores promueven el incumplimiento de estándares mínimos de calidad que afectan directamente la seguridad física y mental de los núcleos familiares, lo que se traduce en inconsistencias de carácter técnico en malas prácticas constructivas, capaces de materializar enfermedades respiratorias, perinatales, cutáneas, pulmonares, estrés, accidentes y aplastamientos (García, García y Torres, 2017).

Por lo anterior, cuando se habla de malas prácticas constructivas se deben abordar dos ópticas, una desde la parte estructural y otra desde la habitabilidad de la vivienda. La primera se relaciona directamente con el no cumplimiento de las exigencias mínimas que garanticen que la vivienda es segura para salvaguardar las vidas humanas, por lo que es importante que la vivienda posea la forma más simétrica posible, tanto en planta, como en su elevación y en sus muros, en búsqueda de una proporción simétrica en cuanto a su distribución, tanto de forma vertical como horizontal según el diseño de espacios. Es importante también construir los muros del segundo piso sobre los del primer nivel para mejorar la estabilidad de la estructura, tener en cuenta que las losas en cada piso estén bien proporcionadas, y asimismo verificar que el largo de la vivienda no sea mayor a tres veces que el ancho (García, García y Torres, 2017).

Finalmente, las malas prácticas constructivas, en relación con la habitabilidad desde el aspecto arquitectónico, contemplan errores técnicos constructivos en la vivienda que interfieren y limitan la calidad frente a su diseño y terminados, los cuales condicionan seguidamente el grado de hacinamiento y salubridad de los moradores frente a los materiales para su edificación, confort y factores físicos y biológicos que forman parte de su entorno inmediato. Cabe resaltar que comprender la importancia de la correcta construcción de los espacios al interior de la vivienda afecta el comportamiento, significado y valoración del habitante, permitiéndole un mejor desarrollo físico, biológico, psicológico y social de la persona, de ahí su estrecha vinculación con el concepto de calidad de vida (García, García y Torres, 2017).

\section{Diseño educativo instruccional}

El diseño instruccional como herramienta para la preparación pedagógica ha sido definido como el proceso sistemático de traducir los principios de aprendizaje en planes de estudio para la generación de materiales y actividades que busquen la instrucción de un individuo en temas específicos. Asimismo, el diseño pedagógico es de vital importancia en la búsqueda de un ámbito educativo ordenado y de calidad, debido a que así se transmiten las metas instruccionales que se deben cumplir al momento de dirigir una propuesta educativa independientemente de su modalidad (Martínez, 2009).

Ahora bien, teniendo en cuenta que los diseños instruccionales son un conjunto de estrategias en las que se basan los enfoques de aprendizaje propuestos por los tutores o facilitadores de un curso (Luzardo, 2010), para el caso del estudio planteado, la propuesta de transferencia de conocimiento se diseñó a partir de un enfoque de educación constructivista, ya que se diseñaron las unidades de aprendizaje a partir de la realidad presente en la comunidad, motivando la creación de significados a partir de experiencias propias y el trabajo en grupo. Por consiguiente, lo modelos constructivistas se enfocan en la creación de herramientas que reflejan los conocimientos tácitos de los individuos y grupos, los cuales parten de las experiencias y realidades que se desarrollan en las comunidades y territorios (Lozano, 2007).

Como fundamento metodológico, se han utilizado otras propuestas educativas y enfoques formativos con el fin de adelantar investigaciones en torno a problemáticas sociales inherentes a los contextos urbanos, promoviendo así la motivación 
y el compromiso de los estudiantes frente a un voluntariado en poblaciones que adelantan acciones mancomunadas para la superación de la pobreza multidimensional; de esta manera, se accede a nuevos conocimientos y saberes que se transfieren y reciben de manera bilateral (estudiante/comunidad) y el conocimiento pasa de uno básico a uno más elaborado durante este proceso de enseñanza/ aprendizaje (Trejos, 2014).

En relación a lo anterior, los estudiantes de Ingeniería Civil, a partir de la problemática expuesta y la situación socioeconómica y educativa de los participantes de la comunidad, reconocen que el conocimiento es el resultado de la interacción del individuo con el medio ambiente que lo rodea; los conocimientos se transforman a partir de los propios esquemas y realidades de la persona, y se relacionan con los contextos producto de la interacción con la comunidad que lo rodean. En consecuencia, el estudiante de Ingeniería debe entablar un diálogo con el docente enfocado en el desarrollo de competencias dirigidas a que el estudiante mejore sus conocimientos a partir de la práctica, sea eficiente en la búsqueda de información, ejerza un pensamiento crítico, esté en la capacidad de resolver problemas de índole social, trabaje de manera colaborativa tanto con sus compañeros de clase como con la comunidad objeto de trabajo, participe en redes de conocimiento, gestione la información en diferentes medios sea de manera oral o escrita y sepa comunicarse de manera asertiva con el objetivo de transmitir el conocimiento producto del estudio de una realidad presente en una población (Torres y Ruge, 2013).

De esta manera, se desarrolló un proceso sistemático que buscó aterrizar la realidad encontrada traducida en las malas practicas constructivas, en planes de estudio para facilitar la transferencia de conocimiento. Por tal motivo, la propuesta educativa se convirtió en un elemento facilitador para acercarse a la comunidad y explicarle de manera coloquial su situación frete a los procesos constructivos de la vivienda (Martínez, 2009). De esta forma, este proceso involucró el desarrollo de materiales y actividades pedagógicas, y luego las pruebas y evaluaciones de las actividades del asistente. De esta manera, el diseño instruccional planteó acciones educativas concretas orientadas a la elaboración de materiales que facilitaran la planeación del curso (Méndez, 2010), por tal motivo, se recurrió a unos pasos generales, cuando se habla de elaboración de un diseño instruccional: a) análisis, b) diseño, c) desarrollo, d) implantación e implementación, y e) evaluación (Lozano, 2007).

\section{METODOLOGÍA}

Respecto a la metodología para identificar las malas prácticas constructivas en habitabilidad en campo, y luego realizar un diseño instruccional enmarcado en la educación constructivista, fue indispensable observar en primera instancia las malas prácticas de construcción que se ligaban a la habitabilidad en una vivienda, para esto se propuso un enfoque de investigación cuantitativa, ya que fue necesario medir estadísticamente la realidad presente de la población y su relación directa con las condiciones de habitabilidad en las que están viviendo. Esto sirvió para establecer las problemáticas instruccionales y los módulos de trabajo del curso propuesto.

La investigación también se abordó desde el punto de vista descriptivo, por cuanto se describieron fenómenos, situaciones, contextos y eventos, ya que dichos estudios buscan especificar las propiedades, las características y los perfiles de personas, grupos, comunidades, procesos, objetos o cualquier otro fenómeno que se someta a un análisis (Hernández, Fernández y Baptista, 2010, p. 102). Para este caso, la Red de cooperación construya seguro y sostenible, a la cual pertenece la Corporación Universitaria Minuto de Dios y las universidades Católica de Colombia, Distrital Francisco José de Caldas, La Salle y Piloto de Colombia, desde sus programas de Ingeniería Civil, identificaron 44 malas prácticas ligadas a la habitabilidad en la vivienda informal. Este trabajo cooperativo propuesto por la red de trabajo estuvo 
apoyado por la Fundación para el Desarrollo Técnico Swisscontact, en el marco del proyecto Construya, iniciativa que busca mejorar las condiciones de calidad de vida a partir de la identificación de malas prácticas constructivas en la vivienda informal, su respectiva transferencia de conocimiento en barrios informales.

Asimismo, el enfoque de estudio seleccionado fue el diseño no experimental; este enfoque permitió observar el fenómeno como se presentó en su contexto natural para posteriormente analizarlo. En este caso se evaluaron las condiciones constructivas relacionadas a la habitabilidad en la vivienda informal en donde participaron 25 familias. Por último, la investigación propuesta también se enmarcó en los diseños de investigación transversal, los cuales se caracterizan por la recolección de datos en un solo momento, en un tiempo único. Su propósito es describir variables y analizar su incidencia e interrelación en un momento dado (Hernández, Fernández y Baptista, 2010).

El estudio se desarrolló en Bogotá, Colombia, en la localidad de Kennedy, barrio Villas de Kennedy. Se escogió este epicentro de acción debido a que existía un acercamiento y trabajo previo por parte de la Red de cooperación con 25 familias del sector, las cuales reconocían la labor del grupo de profesionales en temas de vivienda y asimismo se caracterizaban por ser líderes que trabajaban en pro del mejoramiento de su calidad de vida y el de su comunidad.

A partir de lo anterior se realizó un muestreo no probabilístico, en donde la elección de los elementos no depende de la probabilidad, sino de las causas relacionadas con las características de la investigación y del interés del investigador (Hernández, Fernández y Baptista, 2010), motivo por el cual en estas 25 familias se encontró el elemento de representatividad de la población necesaria para la investigación propuesta. Esta pequeña porción de la comunidad cumplía con las características necesarias que rodean a la vivienda informal con bajo desarrollo constructivo en habitabilidad, alta vulnerabilidad socioeconómica por su estratificación y la iniciativa de querer capacitarse para mejorar sus condiciones de habitabilidad.

Una vez se seleccionó la muestra, se presentó una herramienta con preguntas cerradas, las cuales relacionaron categorías u opciones de respuesta que fueron previamente delimitadas; es decir, a los participantes se les presentaron las posibilidades de respuesta, quienes se acotaron a estas. Además, docentes y estudiantes de ingeniería pertenecientes a la Red de cooperación se aseguraron que los participantes a los cuales se les administró el instrumento conocían y comprendían las categorías de respuesta. Siempre que se pretenda efectuar un análisis estadístico de la información obtenida, se requiere codificar las respuestas de los participantes a las preguntas del cuestionario, por eso fue necesario asignarles símbolos o valores numéricos (Hernández, Fernández y Baptista, 2010).

En consecuencia, se propuso para el estudio la aplicación de una herramienta como la encuesta, la cual se usa principalmente para conocer la opinión de las personas respecto a una variedad de temas, además es la modalidad típica de recolección de la investigación cuantitativa y la forma más común de obtención de datos empíricos de los estudios cuantitativos, por lo que se utilizó dicho instrumento para indagar acerca de las malas prácticas constructivas en habitabilidad en la vivienda informal (Sylvain y Ginette, 2008).

Luego de surtir el proceso anteriormente descrito se realizaron los nueve pasos basados en el modelo instruccional de Jerrold y Kemp (según Lozano (2007), para diseñar un curso, en donde se tuvo el rigor metodológico para diseñar la propuesta educativa enmarcada en las 44 malas prácticas constructivas en habitabilidad. Esto fue diseñado con estudiantes y docentes de la Red:

1. Identificar los problemas instruccionales y especificar los objetivos para los que se diseña el programa instruccional.

2. Examinar aquellas características del estudiante que deben recibir atención durante la planificación. 
3. Identificar claramente los contenidos y analizar los componentes relacionados con los propósitos y objetivos de la instrucción.

4. Elaborar una lista de objetivos instruccionales que deben ser comunicados al estudiante.

5. Secuenciar los contenidos en unidades didácticas y que respondan a un aprendizaje lógico.

6. Diseñar estrategias didácticas que permitan que el estudiante alcance los objetivos que se le proponen.

7. Planificar las estrategias de carácter didáctico.

8. Diseñar instrumentos de evaluación para determinar si se cumplieron los objetivos de la instrucción en el estudiante.

9. Seleccionar los recursos que soportan la instrucción y las actividades didácticas propuestas.

Después de tener la información a transmitir a la comunidad enmarcada en una propuesta de curso, se aplicó la metodología de formación social participativa, la cual comprendió cuatro etapas dinámicas, flexibles y pertinentes a las necesidades profesionales y sociales de los participantes en este tipo de escenarios socioeconómicos; asimismo dicha metodología permitió acercar al estudiante de Ingeniería Civil al trabajo comunitario. Las etapas funcionaron de la siguiente manera:

- Etapa 1. Reconocimiento y análisis del escenario del problema. Se invitó a los estudiantes, docentes y comunidades participantes a acercarse a la historia y evolución del escenario sobre el cual se intervendría. Este reconocimiento del escenario problema se dio a partir de técnicas participativas, como ejercicios de cartografía social, grupos focales, interpretación de imágenes y símbolos. Durante esta etapa los participantes del proyecto aprendieron a reconocerse como sujetos históricos, anclados a un territorio en particular y por tanto responsables de este.

- Etapa 2. Identificación y potencialización de las habilidades y los recursos. Se identificaron las habilidades y potencialidades de las personas; es decir, se buscó articular los saberes, experiencias y conocimientos científicos y populares para que se generaran diversas lecturas de las situaciones problema y así generar perfiles de liderazgo y organización de equipos de trabajo con responsabilidades y roles específicos.

- Etapa 3. Puesta en marcha de las ideas. El objetivo de esta etapa fue convertir las hipótesis de trabajo en acciones concretas. Esta parte fue crucial en el desarrollo de la metodología y en sí del proyecto, pues la articulación de los diversos saberes, los recursos, el tiempo y las tareas, permitieron el desarrollo de la propuesta educativa.

- Etapa 4. Transformación social del entorno. Se reflexionó si los propósitos y objetivos de la propuesta educativa se lograron y si estos fueron concertados, aceptados y satisfactorios por $y$ para los participantes.

En definitiva, el compromiso con las comunidades, las necesidades sentidas identificadas, los objetivos comunes y la incorporación de nuevos conocimientos y experiencias a partir de una realidad presente, son aspectos clave para el desarrollo de una práctica comunitaria que posibilita la apertura al diálogo en un escenario local y didáctico en el cual se guía, propone y no se imponen las ideas, abriendo paso al intercambio, la gestión y la diversidad del conocimiento (Guevara, González y Cavanzo, 2017).

Así, la metodología de formación social participativa facilitó, por un lado, que los estudiantes de Ingeniería Civil involucrados en el proyecto se percibieran como aquellos profesionales que, con un conocimiento multidisciplinar y un estudio previo del contexto como el que intervinieron, están en condiciones de aportarle al presente y pensar el futuro a partir de iniciativas sociales que tengan como apuesta fundamental la construcción colectiva del conocimiento. Por otro, la comunidad comprendió que la percepción del entorno es diferente para cada uno y, por ello, se deben aceptar las diferencias y trabajar de manera conjunta a partir de ellas. 


\section{RESULTADOS}

Los anteriores procesos metodológicos permitieron la identificación en campo de las malas prácticas constructivas (tabla 1). Luego, se analizó la información obtenida, y así se determinó el grado de cumplimiento de las malas prácticas constructivas presentes en las viviendas de la comunidad con el propósito de identificar de primera mano las problemáticas instruccionales que requerían especial atención para el desarrollo del curso propuesto.

Tabla 1. Malas prácticas constructivas de habitabilidad en la vivienda informal

\begin{tabular}{|c|c|c|c|}
\hline $\begin{array}{l}\text { 1. Instalar deficientemente } \\
\text { marcos de ventanas y puertas. }\end{array}$ & $\begin{array}{l}\text { 2. No construir dispositivos } \\
\text { de aprovechamiento de luz } \\
\text { natural. }\end{array}$ & $\begin{array}{l}\text { 3. No construir pasamanos en } \\
\text { escaleras. }\end{array}$ & $\begin{array}{l}\text { 4. No instalar puntos hidráu- } \\
\text { licos. }\end{array}$ \\
\hline $\begin{array}{l}\text { 5. No construir alfajías en } \\
\text { vanos. }\end{array}$ & $\begin{array}{l}\text { 6. Ubicación inadecuada de } \\
\text { luminarias. }\end{array}$ & $\begin{array}{l}\text { 7. No usar mortero de pega } \\
\text { en las juntas verticales }\end{array}$ & $\begin{array}{l}\text { 8. Usar diámetros menores } \\
\text { para evacuación de agua } \\
\text { lluvia. }\end{array}$ \\
\hline $\begin{array}{l}\text { 9. No construir cortagoteras } \\
\text { en aleros de losas y vanos } \\
\text { profundos. }\end{array}$ & $\begin{array}{l}\text { 10. Uso de luminarias insu- } \\
\text { ficientes en espacios funcio- } \\
\text { nales. }\end{array}$ & $\begin{array}{l}\text { 11. Separar la cubierta de la } \\
\text { viga de amarre superior. }\end{array}$ & $\begin{array}{l}\text { 12. Usar diámetros menores } \\
\text { en tuberías para evacuación } \\
\text { de aguas servidas. }\end{array}$ \\
\hline $\begin{array}{l}\text { 13. No construir filtros para } \\
\text { el manejo de agua lluvia en } \\
\text { sistemas de contención de } \\
\text { tierras. }\end{array}$ & $\begin{array}{l}\text { 14. Uso de pinturas o recubri- } \\
\text { mientos brillantes al interior } \\
\text { de la vivienda. }\end{array}$ & $\begin{array}{l}\text { 15. No construir muro, } \\
\text { medianero o posterior, de } \\
\text { separación entre predios } \\
\text { colindantes. }\end{array}$ & $\begin{array}{l}\text { 16. Enchapes porosos o } \\
\text { carencia de estos en cocinas } \\
\text { y baños. }\end{array}$ \\
\hline $\begin{array}{l}\text { 17. No impermeabilizar los } \\
\text { elementos de cimentaciones y } \\
\text { losas de contrapiso. }\end{array}$ & $\begin{array}{l}\text { 18. No construir muebles } \\
\text { fijos para almacenamiento, en } \\
\text { alcobas, cocina y baño. }\end{array}$ & $\begin{array}{l}\text { 19. Conductores eléctricos } \\
\text { descubiertos y en mal estado. }\end{array}$ & $\begin{array}{l}\text { 20. Usar materiales rústicos o } \\
\text { deslizantes en escaleras. }\end{array}$ \\
\hline $\begin{array}{l}\text { 21. No impermeabilizar losas } \\
\text { de cubierta. }\end{array}$ & $\begin{array}{l}\text { 22. Baja funcionalidad de la } \\
\text { vivienda por distribución de } \\
\text { espacios. }\end{array}$ & $\begin{array}{l}\text { 23. Instalar circuitos insufi- } \\
\text { cientes o mala distribución de } \\
\text { la carga eléctrica. }\end{array}$ & $\begin{array}{l}\text { 24. Construir cubiertas livia- } \\
\text { nas con diferentes materiales. }\end{array}$ \\
\hline $\begin{array}{l}25 \text {. No instalar flanches en } \\
\text { remates sobre muros o contra- } \\
\text { muros superiores, inferiores o } \\
\text { laterales. }\end{array}$ & $\begin{array}{l}\text { 26. No construir patio / No } \\
\text { instalar ventilación y reventi- } \\
\text { lación en redes sanitarias. }\end{array}$ & $\begin{array}{l}\text { 27. No instalar cajas eléc- } \\
\text { tricas y canalizaciones para } \\
\text { conductores eléctricos. }\end{array}$ & $\begin{array}{l}\text { 28. Construir cubiertas livia- } \\
\text { nas con pendiente y traslapos } \\
\text { insuficientes. }\end{array}$ \\
\hline $\begin{array}{l}\text { 29. Utilizar canales de reco- } \\
\text { lección y conducción de agua } \\
\text { lluvia con baja pendiente }\end{array}$ & $\begin{array}{l}\text { 30. Anclar deficientemente las } \\
\text { barandas de escaleras. }\end{array}$ & $\begin{array}{l}\text { 31. Cajas de inspección sin } \\
\text { tapa. }\end{array}$ & $\begin{array}{l}\text { 32. Construir losas de cubierta } \\
\text { mal niveladas o sin pendiente } \\
\text { suficiente. }\end{array}$ \\
\hline $\begin{array}{l}\text { 33. No separar zonas húme- } \\
\text { das con registros o válvulas de } \\
\text { cierre hidráulico. }\end{array}$ & $\begin{array}{l}\text { 34. Construir divisiones altas } \\
\text { en las duchas o no construir- } \\
\text { las. }\end{array}$ & $\begin{array}{l}\text { 35. Conectar red de aguas } \\
\text { residuales al alcantarillado de } \\
\text { aguas lluvias. }\end{array}$ & $\begin{array}{l}\text { 36. Mala ejecución de ama- } \\
\text { rres de tejas a los elementos } \\
\text { de soporte. }\end{array}$ \\
\hline $\begin{array}{l}\text { 37. Carencia de sifones en } \\
\text { salidas sanitarias. }\end{array}$ & $\begin{array}{l}\text { 38. Construir barandas con } \\
\text { mucha distancia entre ele- } \\
\text { mentos. }\end{array}$ & $\begin{array}{l}\text { 39. Mezclar aguas lluvias y } \\
\text { aguas servidas en las redes } \\
\text { internas. }\end{array}$ & $\begin{array}{l}\text { 40. No dejar ventanas o duc- } \\
\text { tos de ventilación. }\end{array}$ \\
\hline $\begin{array}{l}\text { 41. Construir filos y vértices } \\
\text { (puntas) en baños, cocinas y } \\
\text { muebles fijos. }\end{array}$ & $\begin{array}{l}\text { 42. Construir escaleras con } \\
\text { peldaños (huella y contrahue- } \\
\text { lla) de diferentes dimensiones. }\end{array}$ & $\begin{array}{l}\text { 43. No construir ductos técni- } \\
\text { cos para instalaciones. }\end{array}$ & $\begin{array}{l}\text { 44. No instalar elementos de } \\
\text { ventilación para evacuación } \\
\text { de gases producto de la que- } \\
\text { ma de combustibles. }\end{array}$ \\
\hline
\end{tabular}

Fuente: Proyecto Construya Fundación Swisscontact.

Los anteriores hallazgos se transfirieron a la comunidad por medio de las unidades de aprendizaje dispuestas en la propuesta educativa constructivista y participativa (tabla 2). A partir de este modelo de curso se pretendió, por un lado, dar respuesta a los interrogantes generados en la investigación y, por otro, a las problemáticas encontradas en la comunidad recabadas durante la fase de 
recolección de datos. De esta manera se brindó, a las poblaciones con una precaria habitabilidad, un entorno educativo que les explicara la importancia de las buenas prácticas constructivas para la salubridad en los espacios de la vivienda. (constructivismo/aporte en ingeniería).

Cabe destacar que desde otros enfoques se han elaborado diversos estudios y metodologías que por un lado reconocen la importancia de la participación de las comunidades para la prevención, investigación y monitoreo de deficiencias en las prácticas constructivas, y por otro, señalan que para el diseño y evaluación de viviendas y sus condiciones de habitabilidad debe reconocerse la existencia de diversas prácticas constructivas que difieren en muchos aspectos de las construcciones formales, lo que se sugiere la necesidad de incluir otras variables en la formulación de dichas prácticas (Porras, Ubaque y Soler, 2017).

Este trabajo aportó a los estudiantes de Ingeniería Civil un escenario social claro en el cual se requirió de compromiso y habilidades comunicacionales y técnicas para responder a las necesidades sentidas de las poblaciones que habitan en condiciones precarias de habitabilidad, como se manifiesta en el marco teórico. Dicha experiencia humanizó el quehacer del estudiante, al ayudarle a comprender la importancia de su ejercicio profesional en los contextos sociales, trabajando a la par con las comunidades, sin imponer o promover procesos asistencialistas que limitaran la transmisión de conocimiento y la sostenibilidad de este tipo de procesos. Asimismo, este ejercicio ayudó a los estudiantes a poner en práctica tanto sus conocimientos técnicos como sociales y así alcanzar logros personales y profesionales en el marco de un trabajo comunitario bilateral.

Con lo anterior se consolidó un espacio común de trabajo en donde los estudiantes, aliados y la comunidad, pudieron dialogar en torno a las malas prácticas constructivas en la vivienda informal y concientizarse acerca de la importancia de la educación con sentido social y su estrecha relación con la salud y sus derechos. Con este espacio se consiguió también transmitir conocimiento y construir un puente entre la teoría y la práctica a través de las unidades de aprendizaje, las cuales permitieron socializar la investigación adelantada por la Red de conocimiento.

Por otra parte, fue posible que los participantes reconocieran sus capacidades individuales y colectivas de trabajo y de la importancia de adquirir

Tabla 2. Modelo de curso enfocado a mitigar las malas prácticas constructivas en habitabilidad

\begin{tabular}{|c|c|c|c|}
\hline Unidades de aprendizaje & Conceptos & Objetivo general & Duración \\
\hline \multirow[b]{2}{*}{ Confort en la vivienda } & Higrotermicidad & \multirow{2}{*}{$\begin{array}{l}\text { Determinar la importancia de la ventilación, } \\
\text { iluminación, humedad y confort en la vivienda, } \\
\text { ligados a diseño en espacios, manejo de aguas } \\
\text { Iluvias, ventanales, claraboyas, baños y cocinas. }\end{array}$} & Tres semanas \\
\hline & Iluminación & & \\
\hline \multirow[b]{2}{*}{ Funcionalidad de la vivienda } & Espacios suficientes & \multirow{2}{*}{$\begin{array}{l}\text { Establecer la importancia de la funcionalidad de } \\
\text { la vivienda a partir del estudio de las instalacio- } \\
\text { nes eléctricas, patio, escaleras, alcobas y red de } \\
\text { aguas residuales. }\end{array}$} & Dos semanas \\
\hline & $\begin{array}{l}\text { Espacios separados y } \\
\text { seguros }\end{array}$ & & \\
\hline $\begin{array}{l}\text { Responsabilidad social y ética } \\
\text { de la vivienda }\end{array}$ & $\begin{array}{l}\text { La vivienda como espa- } \\
\text { cio vital y social. }\end{array}$ & $\begin{array}{l}\text { Sensibilizar frente a los impactos sociales que } \\
\text { generan los diversos procesos constructivos } \\
\text { informales y cómo estos alteran las dinámicas de } \\
\text { las familias y las comunidades. }\end{array}$ & Dos semanas \\
\hline Terminados de la vivienda & $\begin{array}{l}\text { Manejo de pisos, pare- } \\
\text { des y techos }\end{array}$ & $\begin{array}{l}\text { Identificar los materiales para edificar la vi- } \\
\text { vienda desde los aspectos de calidad y proceso } \\
\text { constructivo. }\end{array}$ & Dos semanas \\
\hline
\end{tabular}

Fuente: elaboración propia. 
conocimientos previos en el marco de vivienda y habitabilidad para mejorar su situación personal y comunitaria. En este sentido, fue importante informar e instruir a las poblaciones sobre los riesgos y la responsabilidad social que implica el construir de manera empírica, y sin ningún conocimiento previo. De esta manera se pudo impulsar a la comunidad a preocuparse por su situación de habitabilidad y el mejoramiento de su calidad de vida, pues se generó un escenario educativo pertinente a sus necesidades y habilidades.

También, cabe mencionar los diversos aprendizajes significativos que desarrollaron los estudiantes de Ingeniería Civil que participaron en el desarrollo de la propuesta educativa (tabla 3 ).

Los aprendizajes significativos surgieron en el marco de diversas estrategias pedagógicas, como: mesas de discusión, círculos de diálogo y talleres de sensibilización social entre otros. Dichas estrategias de enseñanza/aprendizaje sirvieron para que tanto los estudiantes de ingeniería como los participantes del proyecto reconocieran que la ingeniería en una profesión dinámica, responsable y con un alto compromiso social, pues está en capacidad de dar respuestas a los múltiples problemas que se presentan en la sociedad -entre ellos, la calidad de la vivienda- y por ello uno de sus retos es estar en continua renovación.

Finalmente, se evidenció por medio de una evaluación que el ejercicio adelantado entre la comunidad y los estudiantes de ingeniería tuvo un resultado positivo debido a que los asistentes pasaron de un conocimiento básico a uno más elaborado al comprender las malas prácticas constructivas y su relación con la calidad de vida. La comunidad cooperó con los estudiantes de ingeniería civil, y los estudiantes se percataron que su investigación tuvo un efecto positivo en la comunidad objeto de estudio.

Tabla 3. Aprendizajes adquiridos

\begin{tabular}{|c|c|c|}
\hline Aprendizajes específicos & Aprendizajes intersociales & Aprendizajes instrumentales \\
\hline $\begin{array}{l}\text { - Situar los problemas en los contex- } \\
\text { tos sociotécnico y operacional. } \\
\text { - Identificar soluciones integrales } \\
\text { a los problemas instruccionales } \\
\text { producto de situaciones cotidianas } \\
\text { para el desarrollo integral de los } \\
\text { individuos. } \\
\text { - Plantear y resolver problemas con } \\
\text { los recursos disponibles. } \\
\text { - Vincular los diversos saberes y } \\
\text { habilidades de las comunidades, } \\
\text { siempre con respeto por sus saberes } \\
\text { y la apropiación del territorio. }\end{array}$ & $\begin{array}{l}\text { - Capacidad analítica y crítica. } \\
\text { - Capacidad para el trabajo colabo- } \\
\text { rativo. } \\
\text { - Habilidad para pensar creativa- e } \\
\text { interdisciplinariamente. } \\
\text { - Capacidad de comunicar y liderar. } \\
\text { - Capacidad de respetar la diversidad } \\
\text { y multiculturalidad de la comunidad. }\end{array}$ & $\begin{array}{l}\text { - Capacidad de observar y analizar problemas } \\
\text { técnicos y sociales en torno a la vivienda infor- } \\
\text { mal. } \\
\text { - Habilidad para observar, percibir y analizar la } \\
\text { realidad de las comunidades y aportar desde los } \\
\text { propios saberes. } \\
\text {-Utilización de herramientas pedagógicas, } \\
\text { codificadas y metodológicas, para entender los } \\
\text { contextos sociales } \\
\text { - Facilidad para involucrarse en la gestión de } \\
\text { proyectos formativos con relación a la vivienda } \\
\text { desde los propios saberes. } \\
\text {-Habilidad para aprender centrado en la experi- } \\
\text { mentación y en la construcción y desconstruc- } \\
\text { ción de saberes científicos. }\end{array}$ \\
\hline
\end{tabular}

Fuente: elaboración propia.

\section{CONCLUSIONES}

Dados los resultados y aprendizajes obtenidos durante la implementación del proyecto, se recomienda seguir las metodologías de carácter formativo nombradas en este documento cuando se piense en abordar, desde el aspecto educativo y participativo, a comunidades que conviven en construcciones y entornos en los que la habitabilidad se ve seriamente comprometida. Esto, debido a las malas prácticas constructivas y el desconocimiento de los riesgos e impactos a la salud física, 
mental y social que acarrea habitar en viviendas cuyo grado de desarrollo informal genera un detrimento en la calidad de vida de sus moradores.

La ocupación desordenada del territorio urbano ha aumentado la informalidad en la obtención de predios, en la construcción de la vivienda, en la utilización de materiales de construcción de desecho o en malas condiciones, y en general, en el aumento de la servidumbre de las unidades habitacionales que se desarrollan progresivamente sin control, promoviendo estructuras inseguras para la habitabilidad de los núcleos familiares que no dimensionan el grado de riesgo físico y mental al que están expuestos.

En el marco de la educación en ingeniería, es importante que las academias, las instituciones $u$ otras iniciativas sociales que se preocupan por mejorar el bienestar y la calidad de vida de las personas, introduzcan en sus programas curriculares la política habitacional. Así no solo se estaría promoviendo un aporte concreto al mejoramiento de los entornos y las condiciones en los que las personas habitan y participan; sino que también se generaría una actitud permanente de aprender a aprender y de aplicación del conocimiento para interpretar y transformar una realidad concreta.

Las orientaciones educativas a nivel constructivistas como la presentada en este trabajo se caracterizan, en su mayoría, por entender que el conocimiento se construye gracias a la participación en comunidades de aprendizaje específicas, dentro de valores y grupos sociales concretos, entendiendo desde la ingeniería que la transferencia de conocimiento es una experiencia compartida, social, más que individual, en donde se debe respetar la cultura y saberes de los demás

Finalmente, una de las claves para mejorar las condiciones de la vivienda es mitigar las malas prácticas constructivas y que las personas comprendan que la vivienda es un elemento esencial para los procesos de la estima personal, familiar y social, además que la calidad de la habitabilidad es un factor crítico para determinar el estado de salud de las poblaciones. En este sentido será importante continuar diseñando propuestas educativas participativas que fortalezcan los vínculos entre la vivienda y el desarrollo humano.

\section{REFERENCIAS}

Asociación Colombiana de Ingeniería Sísmica (2001). Manual de construcción, evaluación y rehabilitación sismorresistente de viviendas de mampostería. Bogotá, Colombia: Red de Estudios Sociales en Prevención de Desastres en América Latina. DOI: https://doi.org/10.18867/ris.79.29

Cubero, R. (2005). Elementos básicos para un constructivismo social. Avances en Psicología Latinoamericana, 23, 43-61. Recuperado de http:// redalyc.uaemex.mx/src/inicio/ArtPdfRed. jsp?iCve $=79902305$

Escallón, C. (2012). La vivienda de interés social en Colombia, principios y retos. Revista de Ingeniería Universidad de los Andes, 35, 55-60.

Espinoza, A. y Gómez, G. (2010). Hacia una concepción sociofísica de la habitabilidad: espacialidad, sustentabilidad y sociedad. Palapa 5(10), 59-69. Recuperado de http://www.redalyc.org/ pdf/948/94820714006.pdf

García, J., García, C. y Torres, C. (2017). Habitabilidad de la vivienda: una perspectiva de salud. 1a. ed. Bogotá, Colombia: Centro Editorial Facultad de Medicina, Universidad Nacional de Colombia. DOI: https://doi.org/10.18273/revsal.v49n2-2017006

Guevara B., J., González G., E. y Cavanzo N., G. (2017). Sistema de gestión del conocimiento para generación de escenarios didácticos para la diversidad. Tecnura, 20, 108-121. DOI: https://doi. org/10.14483/22487638.11683

Hernández, R., Fernández, C. y Baptista, P. (2006). Metodología de la investigación. 5a. ed. Iztapalapa, México: McGraw-Hill Interamericana.

Lozano, A. (2007). Actualidades del diseño instruccional. En Desarrollo del Nuevo Perfil Docente (pp. 35-54). México: Tecnológico de Monterrey.

Luzardo, H. (2019). Modelos de diseño instruccional. Recuperado de: https://es.scribd.com/ document/327224774/modelos-di-pdf 
Martínez, A. (2009). El diseño instruccional en la educación a distancia, un acercamiento a los modelos. Apertura, 9(10), 104-119.

Morán, A. (2010). Un modelo de formación ciudadana. Soporte de procesos de transformación social. Revista Prospectiva, 15, 105-133. Cali, Colombia: Universidad del Valle. DOI: https://doi. org/10.25100/prts.v0i15.1107

Pontificia Universidad Javeriana (2012). Identificación de las principales amenazas para la salud física y mental de las poblaciones que habitan en sectores urbanos de origen informal de Bogotá. Bogotá, Colombia: Instituto Javeriano de Vivienda y Urbanismo, Facultad de Arquitectura y Diseño e Instituto de Promoción de la Salud, Facultad de Medicina. DOI: https://doi.org/10.18273/revsal. v49n2-2017001

ONU-Hábitat (2014). Construcción de ciudades más equitativas. Recuperado de http://publicaciones. caf.com/media/39869/construccion_de_ciudades_ mas_equitativas_web0804.pdf

Rueda, N. (2011). La integración de la demanda informal a la política de vivienda: algunas opciones iniciales. Revista de Ingeniería Universidad de los Andes, 35, 108-115.

Sylvain, G. y Ginette T. (2008). Métodos y técnicas de muestreo. En Metodología de las ciencias humanas (pp. 93-127) Distrito Federal, México: Fondo del Cultura Económica.

Torres, C. y Ruge, J. (2013). Gestores comunitarios del hábitat: una propuesta educativa desde la ingeniería civil para mejorar los problemas de habitabilidad de comunidades marginales. (Caso barrio Villa del Cerro. Bogotá, Colombia). Revista Educación en Ingeniería, 8(16), 125-135. Recuperado de https:// www.educacioneningenieria.org/index.php/edi/article/view/309/180. DOI: https://doi.org/10.1344/ ridu2019.11.10

Trejos Buritica, O.I. (2014). Relaciones de aprendizaje significativo entre dos paradigmas de programación a partir de dos lenguajes de programación. Tecnura, 18, 91-102. DOI: 10.14483/udistrital.jour.tecnura.2014.3a07. DOI: https://doi.org/10.14483/ udistrital.jour.tecnura.2014.3.a07

Zulaica, L. y Celemín, J. (2008). Análisis territorial de las condiciones de habitabilidad en el periurbano de la ciudad de Mar del Plata (Argentina), a partir de la construcción de un índice y de la aplicación de métodos de asociación espacial. Revista de Geografía Norte Grande, 41, 129-146. Recuperado de http://www.scielo.cl/ scielo.php?pid=S0718-4022008000300007 \&script=sci_arttext. DOI: https://doi.org/10.4067/ s0718-34022008000300007

\section{(c) (1) () (2)}

